\title{
Performance Comparison of Different Types of Receivers in Indoor MIMO-VLC Systems
}

\section{Chen CHEN and Wen-De ZHONG ${ }^{*}$}

\author{
School of Electrical and Electronic Engineering, Nanyang Technological University \\ 50 Nanyang Avenue, Singapore 639798 \\ *Email: ewdzhong@ntu.edu.sg
}

Keywords: Visible light communication, Multiple-input multiple-output, Area spectral efficiency.

\begin{abstract}
In this paper, we compare the performance of non-imaging receiver (NImR), imaging receiver (ImR) and imaging angle diversity receiver (ImADR) in an indoor multiple-input multiple-output visible light communication (MIMO-VLC) system, in terms of area spectral efficiency (ASE). Analytical ASE expressions of MIMO-VLC systems using different types of receivers are derived and our analysis show that the MIMO-VLC system using an ImR or an ImADR achieves significantly improved ASE performance compared with the system using a NImR.
\end{abstract}

\section{Introduction}

As a promising alternative and complementary technology to RF technology, white light-emitting diodes (LEDs) enabled visible light communication (VLC) has attracted great interest for providing high-speed and electromagnetic interference-free wireless communication in indoor environments [1]. In order to provide sufficient illumination, multiple LEDs are commonly mounted in the ceiling. By leveraging the existing LED lighting fixtures, it is very natural to employ multiple-input multiple-output (MIMO) transmission to increase the capacity of VLC systems [2,3]. Three types of receivers can be applied in indoor MIMO-VLC systems, including non-imaging receiver (NImR), imaging receiver (ImR) and imaging angle diversity receiver (ImADR) [2-4].

So far, a few comparisons between NImR and ImR in indoor MIMO-VLC systems have been reported in the literature [2,5]. However, the comparisons are performed in terms of bit error rate (BER) for a specific link. Considering that a MIMO-VLC system always covers a certain area in an indoor environment, it is necessary and of great significance to investigate the system performance over its coverage. The area spectral efficiency (ASE), which was previously proposed for cellular mobile radio system as a suitable measure of spectral efficiency over a certain area [6], can be an effective performance metric for comparing different receivers in indoor MIMO-VLC systems.

In this paper, we compare the ASE performance of NImR, ImR and ImADR in an indoor $4 \times 4$ MIMO-VLC system. Analytical ASE expressions of an indoor MIMO-VLC system using NImR, ImR, and ImADR are derived. The obtained analytical results show that the ASE performance of the $4 \times 4$ MIMO-VLC system can be significantly enhanced when an ImR or an ImADR is used instead of a NImR. The impact of different spacings between two adjacent LEDs and the number of active users on the ASE performance of the system in a $5 \mathrm{~m} \times 5 \mathrm{~m} \times 3 \mathrm{~m}$ room is also analyzed. 

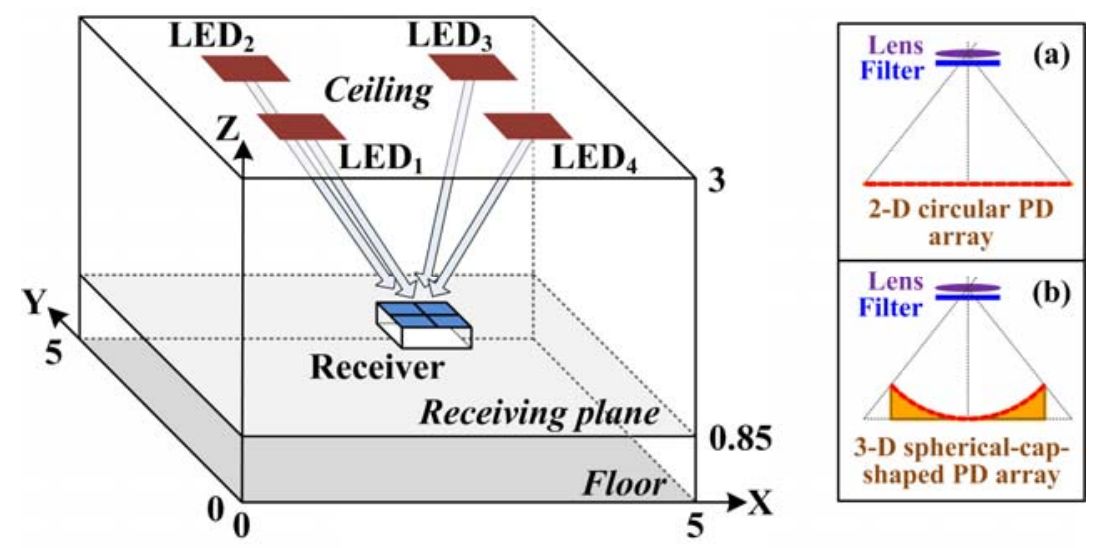

Figure 1 . Geometric setup of an indoor $4 \times 4$ MIMO-VLC system. Insets: side views of (a) a conventional ImR and (b) an ImADR.

\section{Indoor MIMO-VLC System Using Different Types of Receivers}

In this section, we describe the model of a general indoor MIMO-VLC system. Without loss of generality, an indoor $4 \times 4$ MIMO-VLC system is considered. Fig. 1 illustrates the geometric setup of the $4 \times 4$ MIMO-VLC system in a typical room. Totally four LEDs are mounted in the ceiling for illumination and data transmission. The radiated light is captured by a receiver which is located at the receiving plane.

Three different types of receivers can be used in the MIMO-VLC system, including NImR, conventional ImR, and ImADR. A NImR is made up of a grid of photodetectors (PDs) and each PD is mounted with an individual concentrator, while an ImR consists of a grid of PDs sharing a common imaging lens [2]. The PDs are vertically oriented in a two-dimensional circular detector array in the conventional $\mathrm{ImR}$, as shown in inset (a) of Fig. 1. However, an ImADR has a three-dimensional spherical-cap-shaped detector array consisting of angle diversity PDs, as shown in inset (b) of Fig. 1. More details about the ImADR can be found in [4].

For an indoor $4 \times 4$ MIMO-VLC system in a typical room with totally $N$ active users, the ASE $\left[\mathrm{bits} / \mathrm{s} / \mathrm{Hz} / \mathrm{m}^{2}\right]$ is defined as the sum of the maximum bit rates per $\mathrm{Hz}$ per unit area which can be approximated by $[6,7]$

$$
A S E=\sum_{k=1}^{N} C_{k} /\left(W A_{\text {room }}\right),
$$

where $C_{k}$ is the maximum data rate (i.e. the Shannon capacity) achieved by the $k^{\text {th }}$ user $(k=1,2, \ldots, N), W$ is the total modulation bandwidth and $A$ room is the area of the room. Assuming that the total modulation bandwidth is equally allocated to $N$ active users, the maximum data rate of the $k^{\text {th }}$ user in the indoor $4 \times 4$ MIMO-VLC system is expressed by

$$
C_{k}=\sum_{i=1}^{4} W_{k} \log _{2}\left(1+S N R_{k i}\right),
$$

where $W_{k}=W / N$ is the bandwidth allocated to the $k^{\text {th }}$ user and $S N R_{k i}$ is the signal-tonoise ratio (SNR) for the $i^{\text {th }}$ channel of the $k^{\text {th }}$ user.

The SNR for the $i^{\text {th }}$ channel of the $k^{\text {th }}$ user in the indoor $4 \times 4$ MIMO-VLC system using a NImR is calculated by Eq. 3 [7]. 


$$
S N R_{k i}^{\mathrm{NImR}}=\frac{\left(R \xi h_{k i} P_{\mathrm{opt}}\right)^{2}}{\sum_{j=1, j \neq i}^{4}\left(R \xi h_{k j} P_{\mathrm{opt}}\right)^{2}+P_{\mathrm{n}}} .
$$

In Eq. $3, R$ is the responsivity of $\mathrm{PD}, \xi$ is the modulation index, $h_{k i}$ is the channel DC gain between the $k^{\text {th }}$ user and the $i^{\text {th }}$ LED, $P_{\text {opt }}$ is the output optical power of the LED, and $P_{\mathrm{n}}$ is the power of the additive noise. The detailed calculation of $P_{\mathrm{n}}$ can be found in [8]. By modeling the LOS irradiance of an LED as a generalized Lambertian radiation pattern, the channel DC gain between the $k^{\text {th }}$ user and the $i^{\text {th }}$ LED is given by

$$
h_{k i}=(m+1) A_{\mathrm{PD}} \mu \eta \cos ^{m}\left(\phi_{k i}\right) \cos \left(\theta_{k i}\right) /\left(2 \pi d_{k i}^{2}\right),
$$

where $m=-\ln 2 / \ln \left(\cos \left(\Psi_{1 / 2}\right)\right)$ is the Lambertian emission order and $\Psi_{1 / 2}$ is the semi-angle at half power of LED, $A$ PD is the active area of $\mathrm{PD}, \mu$ and $\eta$ are the gains of optical filter and lens, respectively, $\phi_{k i}$ is the emission angle, $\theta_{k i}$ is the incident angle, and $d_{k i}$ is the distance. Note that if $\theta_{k i}$ is outside the receiver's field-of-view (FOV), $h_{k i}$ becomes zero.

When a conventional ImR is used, the inter-channel interference (ICI) is negligible and hence the SNR becomes

$$
S N R_{k i}^{\operatorname{ImR}}=\left(R \xi h_{k i} P_{\mathrm{opt}}\right)^{2} / P_{\mathrm{n}} \text {. }
$$

When the ImR is replaced by an ImADR, the incident angle $\theta_{k i}$ is always $0^{\circ}$ and the channel DC gain is improved which is given by [4]

$$
h_{k i}^{*}=(m+1) A_{\mathrm{PD}} \mu \eta \cos ^{m}\left(\phi_{k i}\right) /\left(2 \pi d_{k i}^{2}\right) \text {, }
$$

and hence the SNR using an ImADR is expressed by

$$
S N R_{k i}^{\mathrm{ImADR}}=\left(R \xi h_{k i}^{*} P_{\mathrm{opt}}\right)^{2} / P_{\mathrm{n}} .
$$

Based on Eqs. 1, 2, 3, 5 and 7, the ASE the indoor $4 \times 4$ MIMO-VLC system employing different types of receivers can be obtained.

\section{System Setup and Numerical Results}

Based on the analysis above, we compare the ASE performance of NImR, ImR and ImADR in an indoor $4 \times 4$ MIMO-VLC system. The geometric setup of the system in a room with a dimension of $5 \mathrm{~m} \times 5 \mathrm{~m} \times 3 \mathrm{~m}$ is illustrated in Fig. 1, and the key parameters of the system are listed in Table 1. The area of the room is $25 \mathrm{~m}^{2}$ and the height of the receiving plane is $0.85 \mathrm{~m}$. The semi-angle at half power of LED is $60^{\circ}$, the output optical power of each LED is $10 \mathrm{~W}$, and the modulation index is 0.3 . The gains of the optical filter and lens are both assumed to be 1 . The FOV of the receiver is $150^{\circ}$ such that the users at any position of the receiving plane can receive the light emitted by all the four LEDs. We assume that the NImR consists of four PDs, and each PD has an active area of $1 \mathrm{~cm}^{2}$ and a responsivity of $1 \mathrm{~A} / \mathrm{W}$. For fair comparison, the ImR and the ImADR are also equipped with the same PDs and the spot of each LED falls entirely on one PD [8]. The modulation bandwidth is $10 \mathrm{MHz}$ and the background current is $58 \mu \mathrm{A}$ due to ambient light. 
Table 1. Key parameters of the system

\begin{tabular}{|c|c|}
\hline Parameter & Value \\
\hline Room dimension & $5[\mathrm{~m}] \times 5[\mathrm{~m}] \times 3[\mathrm{~m}]$ \\
\hline Area of the room & $25\left[\mathrm{~m}^{2}\right]$ \\
\hline Height of the receiving plane & $0.85[\mathrm{~m}]$ \\
\hline Semi-angle at half power of LED & $60^{\circ}$ \\
\hline Output optical power of LED & $10[\mathrm{~W}]$ \\
\hline Modulation index & 0.3 \\
\hline Gain of optical filter & 1 \\
\hline Gain of optical lens & 1 \\
\hline FOV of detector & $150^{\circ}$ \\
\hline Active area of PD & $1\left[\mathrm{~cm}{ }^{2}\right]$ \\
\hline Responsivity of PD & $1[\mathrm{~A} / \mathrm{W}]$ \\
\hline Modulation bandwidth & $10[\mathrm{MHz}]$ \\
\hline Background current & $58[\mu \mathrm{A}]$ \\
\hline
\end{tabular}

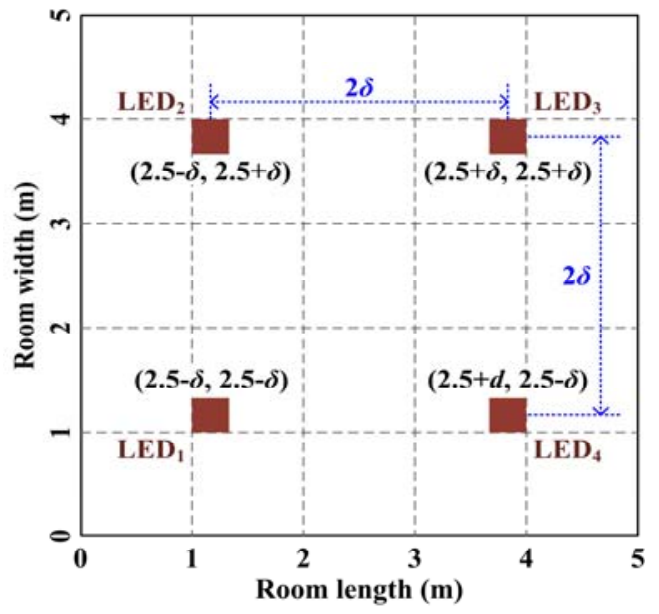

Figure 2. Placement of four LEDs in the ceiling.

The placement of four LEDs in the ceiling is shown in Fig. 2. As we can see, the positions of four LEDs are $(2.5-\delta, 2.5-\delta),(2.5+\delta, 2.5-\delta),(2.5+\delta, 2.5+\delta)$ and $(2.5-\delta$, $2.5+\delta$ ) and the unit is meter. Therefore, the spacing between two adjacent LEDs is given by $2 \delta$. Moreover, totally $N$ active users are assumed to be uniformly distributed in the receiving plane of the room for ASE calculation. In our following analysis, we choose a set of $N$ values as $N=\{4,9,16,25,36,49,64,81\}$.

We first examine the ASE performance of the indoor non-imaging $4 \times 4$ MIMO-VLC system for different spacings between two adjacent PDs in the NImR, where the spacing between two adjacent LEDs is $2 \mathrm{~m}$ and 36 active users are uniformly distributed around the receiving plane. As shown in Fig. 3, the ASE continuously increases as the spacing between two adjacent PDs in the NImR is increased from 1 to $10 \mathrm{~cm}$. The maximum ASE is about $0.9 \mathrm{bits} / \mathrm{s} / \mathrm{Hz} / \mathrm{m}^{2}$ when the spacing is $10 \mathrm{~cm}$. The reason the achievable ASE is very limited is that indoor non-imaging MIMO-VLC systems suffer from severe ICI and hence the SNR is significantly degraded $[2,5]$. Although increasing the spacing between two adjacent PDs can improve the ASE 
performance, the size of the receiver is increased accordingly. In practical applications, the receiver size cannot be too large.

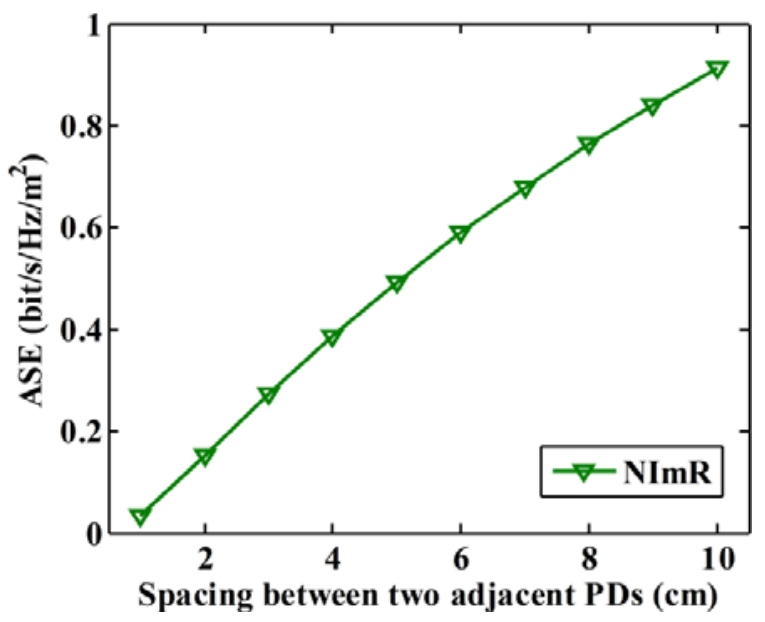

Figure 3. ASE vs. spacing between two adjacent PDs using a NImR.
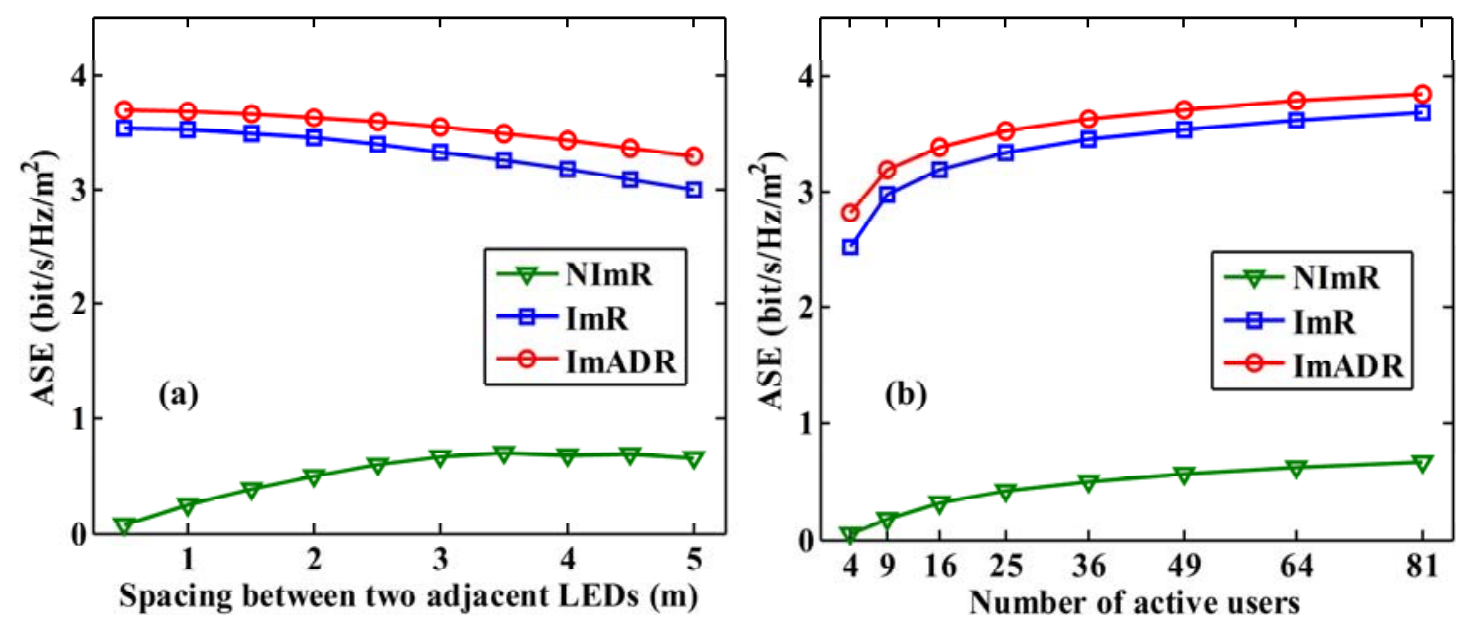

Figure 4. (a) ASE vs. spacing between two adjacent LEDs and (b) ASE vs. number of uniformly distributed active users around the receiving plane.

Therefore, as a trade-off between the ASE performance and the receiver size, a spacing of $5 \mathrm{~cm}$ between two adjacent PDs in the NImR is considered for the following analysis and comparisons.

The relationship between the ASE performance and the spacing between two adjacent LEDs in the ceiling is further investigated. Fig. 4(a) shows the ASE versus the spacing between two adjacent LEDs, where the number of uniformly distributed active users is 36 . It can be clearly seen that the indoor $4 \times 4$ MIMO-VLC system using a NImR can only achieve very limited ASE due to the severe ICI. It can also be observed that the ASE of the indoor $4 \times 4$ MIMO-VLC system using a NImR increases with the increase of the spacing between two adjacent LEDs in the ceiling. Moreover, the maximum ASE of about $0.7 \mathrm{bits} / \mathrm{s} / \mathrm{Hz} / \mathrm{m}^{2}$ is achieved when the spacing is $3.5 \mathrm{~m}$. In contrast, the ASE of the indoor $4 \times 4$ MIMO-VLC system is significantly improved when an ImR is applied and a further ASE improvement can be achieved when an ImADR is employed. For example, when the spacing is $2 \mathrm{~m}$, the ASEs achieved by the indoor $4 \times 4$ MIMO-VLC system using an ImR and an ImADR are 3.4 and 3.6 $\mathrm{bit} / \mathrm{s} / \mathrm{Hz} / \mathrm{m}^{2}$, respectively. Therefore, an ASE improvement of $6 \%$ is achieved by 
employing ImADR instead of ImR. As we can see, the ASE decreases slowly with the increase of the spacing between two adjacent LEDs when ImR or ImADR is utilized. In addition, the ASE improvement is gradually increased with the increase of the spacing. When the spacing is $5 \mathrm{~m}$, i.e., the LEDs are located at the corners of the room, the ASE improvement is $\sim 14 \%$.

The impact of the number of uniformly distributed active users around the receiving plane on the ASE performance of the indoor $4 \times 4$ MIMO-VLC system is also analyzed. Fig. 4(b) shows the ASE versus the number of uniformly distributed active users, where the spacing between two adjacent LEDs is $2 \mathrm{~m}$. The same trend can be found for different types of receivers that the ASE increases with the increase of the number of uniformly distributed active users around the receiving plane.

\section{Summary}

We have analytically compared the ASE performance of an indoor $4 \times 4$ MIMO-VLC system using different types of receivers, i.e. NImR, ImR, and ImADR. The obtained analytical results have shown that the ASE of the indoor $4 \times 4$ MIMO-VLC system using a NImR is very limited due to the severe ICI, and the ASE can be significantly improved when an ImR or an ImADR is applied. Moreover, up to $14 \%$ ASE improvement can be achieved by replacing the conventional ImR with an ImADR.

\section{Acknowledgement}

This work was supported by MOE/NTU AcRF Tier 1 Grant RG 85/13.

\section{References}

[1] H. Haas, Visible light communication, OFC (2015) Tu2G.5.

[2] L. Zeng, D. O’Brien, H. Minh, G. Faulkner, K. Lee, D. Jung, Y. Oh, E. Won, High data rate multiple input multiple output (MIMO) optical wireless communications using white LED lighting, IEEE J. Sel. Areas Commun. 27 (2009) 1654-1662.

[3] C. Chen, W. Zhong, D. Wu, Non-Hermitian symmetry OFDM for MIMO visible light communications, accepted by IEEE/OSA J. Opt. Commun. Netw. (2017).

[4] C. Chen, W. Zhong, D. Wu, Z. Ghassemlooy, Wide-FOV and high-gain imaging angle diversity receiver for indoor SDM-VLC systems, IEEE Photon. Technol. Lett. 28 (2016) 2078-2081.

[5] P. Butala, H. Elgala, and T. Little, "Performance of optical spatial modulation and spatial multiplexing with imaging receiver," IEEE WCNC (2014) 394-399.

[6] M.-S. Alouini, A.J. Goldsmith, Area spectral efficiency of cellular mobile radio systems, IEEE Trans. Veh. Technol. 48 (1999) 1047-1066.

[7] I. Stefan, H. Burchardt, H. Haas, Area spectral efficiency performance comparison between VLC and RF femtocell networks, IEEE ICC (2013) 3825-3829.

[8] C. Chen, W. Zhong, D. Wu, Communication coverage improvement of indoor SDM-VLC system using NHS-OFDM with a modified imaging receiver, IEEE ICC Workshops (2016) 315-320. 